\title{
CASO DE ESTUDIO: FORMATOS COMERCIALES Y SUS EFECTOS EN LAS TIENDAS EN EL BARRIO MANGA, CARTAGENA DE INDIAS*
}

\section{Case study: Supermarket formats and their effects in convenient stores in Manga, Cartagena de Indias}

\section{Laura Cristina Espinosa Flórez ${ }^{1}$ Camila Fernanda Safar Cano ${ }^{1}$ Daniela Licona Dager ${ }^{1}$ Francisco Javier Maza Ávila²}

\section{RESUMEN}

El objetivo de este documento, de carácter explicativo-propositivo, consiste en analizar las apreciaciones que tienen los tenderos del barrio Manga de Cartagena de Indias sobre los efectos que les ha generado la llegada de los formatos comerciales Hard Discount y Express, así como las estrategias que han implementado para mantener su competitividad, como base para la formulación de recomendaciones asegurar su permanencia. Para ello se aplicaron cuestionarios al total de las tiendas ubicadas en el barrio Manga (22), para indagar las características de las tiendas, sus apreciaciones sobre los formatos comerciales y las estrategias implementadas. Los resultados muestran que, los tenderos saben que los formatos comerciales son su competencia, aunque no los consideran un riesgo real para la supervivencia de sus negocios, pues tienen como ventaja, el largo tiempo de establecidas, la cercanía con sus clientes, el significado cultural que representan. De allí las casi nulas estrategias implementadas para enfrentarlos.

Palabras clave: Tienda de barrio, Mini-market, Hard Discount, Express, Percepción.

\footnotetext{
* Documento presentado en las Jornadas de Investigación de la Facultad de Ciencias Económicas de la Universidad de Cartagena

${ }^{1}$ Estudiante del programa de Administración Industrial de la Universidad de Cartagena. Miembro del semillero GIDER. E-mail: lespinosaf@unicartagena.edu.co; csafarc@unicartagena.edu.co; dliconad@unicartagena.edu.co

${ }^{2}$ Doctor en Ciencias Sociales y Jurídicas. Profesor adscrito al programa de Administración Industrial de la Facultad de Ciencias Económicas y al Instituto Internacional de Estudios del Caribe, Universidad de Cartagena. Director del Grupo de Investigación en estudios para el Desarrollo Regional -GIDER-. E-mail: fmazaa@unicartagena.edu.co
} 


\begin{abstract}
The objective of this article is to analyze the perceptions of shopkeepers in the Manga neighborhoods of Cartagena de Indias on the effects that underlie the arrival of the new hard discount and express commercial formats to the sector from the characterization of the stores, the study of the appreciations proper as well as the strategies they say to implement to compete in the market and finally the formulation of specific recommendations to ensure their permanence. For its development, we worked with primary information, through the application of surveys in different establishments, and was supported by secondary information in previous research documents to support the theme of neighborhood shops and new retail chains. Most stores have a large number of established years in the neighborhood, this is justified for the cultural significance they represent and the special way in which they sell their products, with respect to the friendly treatment with their customers and products of first need that offers.
\end{abstract}

Keywords: Shop neighborhood, mini-market, commercial format, hard discount, express commercial format, perception.

\title{
Introducción
}

Los almacenes de cadena, en búsqueda de mayor dominio del mercado, han encontrado la manera más cómoda de llegar al hogar de las personas ampliando su portafolio de servicio mediante los minimarkets. Esta modalidad de ventas al por menor tiene cierta incidencia en las tiendas barriales, las cuales durante años han provisto a los consumidores de productos de la canasta familiar y de primera necesidad. Con su posicionamiento y el paso de los años, las plataformas de distribución tanto nacionales como internacionales también han llegado al barrio de Manga, Cartagena de Indias, en formatos Hard Discount y Express, convirtiéndose entonces, en la competencia directa de la tienda, como canal de distribución tradicional.

La entrada y crecimiento de las cadenas de supermercados, fue vista como una gran amenaza para las tiendas de barrio, que se creía no podían competir con las economías de escala, la variedad de productos, la variedad de marcas y los precios bajos que éstos ofrecían. A pesar de las predicciones realizadas y de las desventajas de las tiendas de barrio frente a los grandes supermercados e incluso mini-markets en el país, los efectos no han sido de la magnitud esperada. (Acevedo Navas, C., y Páramo Morales, 2008). Aun así, estas mantienen una continua disputa con las nuevas alternativas de 
servicios como lo son los formatos comerciales de descuento duro y express. Mientras las grandes superficies en Colombia como el Grupo Éxito, Carulla, la nueva cadena de almacenes ARA, entre otras, procuran diseñar nuevas plataformas más cercanas a los clientes, el formato tradicional con la existencia de factores estratégicos ha permitido que las tiendas de barrio continúen manteniendo una fortaleza a nivel económico, cultural y comercial en Colombia (Tovar Espitia y Mendoza Gómez, 2009). Para el caso particular del barrio Manga en Cartagena, los residentes cuentan con almacenes Éxito y un Carulla, bajo el formato Express, dentro de su zona de confort, además de otras plataformas nuevas, como las tiendas ARA, en formato Hard Discount, que al ubicarse en los alrededores del barrio y dentro del barrio mismo, se convierten de igual manera, en potenciales competidores del negocio.

Este artículo pretende mostrar la apreciación de los tenderos del barrio Manga sobre los efectos que les ha traído la llegada de los nuevos formatos comerciales Hard Discount y Express al barrio Manga de Cartagena de Indias y las estrategias que han implementado para enfrentar dichos efectos, a partir de los resultados de la aplicación de cuestionarios efectuados a la totalidad de tiendas (22) presentes en el mismo barrio, no solo como proveedoras de productos de primera necesidad o de la canasta familiar, sino como parte de la cultura y esencia Cartagenera. En este sentido, la importancia de esta investigación radica en la contribución para que las tiendas del barrio Manga de Cartagena de Indias puedan implementar estrategias que fortalezcan la calidad del servicio que ofrecen, y que logren, a su vez, mantener la permanencia de sus negocios. También se espera que la investigación pueda servir como referencia para estudiar dicho fenómeno en otros barrios en la ciudad.

\section{Marco Teórico- Empírico}

\section{La Tienda}

Se pueden definir como negocios microempresariales que generalmente son desarrollados por un grupo familiar y de muy pequeña escala, en el cual se expenden artículos de primera necesidad, comestibles, bebidas, licores, miscelánea y productos de aseo (Pinilla, O. y González, 2004). Concebidos como pequeños locales diseminados a lo largo y ancho de la geografía nacional, las tiendas guardan particulares característicos, heredadas muy seguramente de sus ancestros comerciales, los tratantes, y su labor más importante es la de expender sus productos al menudeo. Generalmente se desarrollan en la residencia de sus propietarios, sea en la sala, en el garaje o en la parte delantera de su habitación, adecuada para atender a sus clientes. En ella trabajan en promedio 2 o 3 personas, casi siempre 
familiares de los propietarios. Sus jornadas de trabajo son extenuantes, dado que abren a muy tempranas horas de la mañana y cierran muy entrada la noche, y en algunos casos laboran las 24 horas del día, debido a que muchos de ellos atienden a sus clientes sin importar la hora (Londoño, E. y Navas, 2005).

\section{Importancia de la tienda}

La cercanía, la miniaturización de las presentaciones de los productos expendidos, el crédito y el trato personalizado (Ramírez, O. y Pachón, 2004) son algunas de las razones más importantes para su permanencia y dinamismo. En ellas se desarrollan prácticas comerciales basadas en la confianza mutua entre tenderos y consumidores. Prácticas que por el proceso de despersonalización experimentado en las denominadas grandes superficies han venido desapareciendo en ciertas capas de la población. En las tiendas aún persiste el fiado confiando solo en la palabra, el regateo, la ñapa, como las más auténticas expresiones de las relaciones establecidas entre tendero y consumidor (Paramo Morales, García Cano, y Arias Escobar, 2011).

\section{La tienda como canal de distribución}

La tienda, claramente cumple algunas de las funciones de los canales de distribución (Tovar Espitia y Mendoza Gómez, 2009), tales como:

- Centralizan decisiones básicas de la comercialización.

- Se convierten en una partida del activo para el fabricante.

- Intervienen en la fijación de precios, aconsejando el más adecuado.

- Tienen una gran información sobre el producto, competencia y mercado.

- Participan activamente en actividades de promoción.

- Posicionan al producto en el lugar que consideran más adecuado.

- Intervienen directa o indirectamente en el servicio post venta.

- Colaboran en la imagen de la empresa.

- Actúan como fuerza de ventas de la fábrica.

- Venden productos en lugares de difícil acceso y no rentables al fabricante. 


\section{Supervivencia Empresarial}

Desde la perspectiva de Audretsch, la supervivencia empresarial representa el número de empresas supervivientes en una industria en un año dado, como un porcentaje del número total de nuevas empresas de una industria, establecidas en ese año (Audretsch, 1991 citado en (Castillo y No, 2014)).

La definición sobre la supervivencia empresarial describe aquellos mecanismos mediante los cuales los factores internos y externos al área geográfica específica, así como las acciones, interacciones y comportamientos de los agentes que residen en el área geográfica inciden en la dinámica de la permanencia de las empresas en un determinado mercado. La demografía empresarial basa el estudio del nacimiento de las empresas, así como del estudio de aquello que ocurre tras su nacimiento en un mercado (permanencia) y de su muerte (para el presente análisis será la salida), para analizar si las funciones de riesgo y funciones de supervivencia cobran relevancia como modelo para explicar este fenómeno (Castillo y No, 2014).

\section{Percepción Desde las Aproximaciones Teóricas}

\section{Percepción}

La percepción es cualquier acto o proceso de conocimiento de objetos o verdades, ya sea mediante la experiencia o por el pensamiento. Esta percepción no está ligada necesariamente a la actividad de los órganos de los sentidos. Es tanto una forma de pensamiento como una conducta inmediata. La percepción es un proceso equiparable a la discriminación, a la diferenciación y a la observación. Es simbólica, es decir, es una conducta que manifiesta una relación abstracta entre el organismo y su ambiente. El símbolo está contenido en la reacción (Bartley, 1969 citado en (Núñez y López, 2012)).

Precisamente a estas definiciones apunta este estudio sobre las apreciaciones del tendero desde su psicología como comerciante y la reacción a un estímulo para predecir el futuro comportamiento de este individuo. La percepción es la interpretación que hacemos de los inputs; es un proceso constructivo, más que un registro mecánico de la realidad. Cuanto más ambiguo sea el input recibido, más diferirán las construcciones e interpretaciones; por ello, el mercadeo debe procurar conocer las interpretaciones actuales y buscar un posicionamiento claro (Cave, 2005 citado en (Núñez y López, 2012)). 


\section{Sociología de la percepción: dupla percepción/sensación}

Frente al reto de desdibujar desde la geografía de los sentidos las duplas cognición y sensación, la percepción puede entenderse como un proceso que implica sentir y recordar, reconocer y asociar (Rodaway, 1994 citado en (Ramos, 2016)). Es por ello que considerar el mutuo condicionamiento de ambas dimensiones -a saber, percepción y sensación- supone asumir que la percepción siempre está mediada, ya que involucra tanto el cuerpo y la mente como la cultura y el ambiente. Por su parte, la percepción, también implica tanto sensaciones como significados: no es solo una reacción física ante los estímulos del mundo, sino una "experiencia significativa" (meaningful experience), ya que se le atribuyen significados en el momento de percibir (Crossley, 1995 citado en (Ramos, 2016)).

\section{Diseño Metodológico}

Se desarrolló un estudio explicativo - propositivo, por medio de la aplicación de encuestas efectuadas a la totalidad de tiendas presentes (22 tiendas) en el barrio Manga de la Ciudad Cartagena de Indias, para la identificación de las apreciaciones pertinentes con respecto a dicha amenaza posible y el suministro de ciertas recomendaciones para que la población en mención asegure la permanencia de sus negocios. En primer lugar, se caracterizaron todas las tiendas que se encuentran ubicadas en el sector de estudio, luego se indagó sobre el conocimiento y las apreciaciones sobre el grado de amenaza que les ha traído la aparición de las nuevas cadenas de almacenes minoristas y las estrategias que están implementando para competir en el mercado. Finalmente, se formularon recomendaciones específicas para contribuir a reforzar sus estrategias de permanencia en el mercado.

\section{Discusión De Resultados}

Actualmente, en el barrio Manga de la Ciudad Cartagena de Indias, hay un total de 22 tiendas, ubicadas en diferentes sectores del mismo. Del total de ellas, solo el 0,05\% ofrece autoservicio a sus clientes, mientras en las demás, el servicio es proporcionado a los clientes por un empleado de la tienda. Sobre la antigüedad de las tiendas, el 36\% de ellas lleva en el barrio de 6 a 10 años de funcionamiento, el $37 \%$ más de 20 años, el 18\% entre 11 a 15 años, y el 9\% entre 16 y 20 años. Ninguna tiene menos de 6 años de existencia. En la observación de las tiendas, se encontró que en su estructura física cuentan con grandes vitrinas que dividen el lugar de trabajo y ubicación de los productos, con la zona de espera de los clientes. Asimismo, en los productos ofrecidos, se encuentran casi todos los que conforman la canasta familiar y algunos que no están en ella. La presentación y precios de ellos varían según su 
tamaño y marca respectivamente. Además, algunas ofrecen servicios adicionales, tales como los domicilios.

Con respecto a la relación con los clientes, más del $80 \%$ de los tenderos consideran que la proximidad de sus tiendas con las viviendas, los precios que manejan, el horario de atención y la variedad en el surtido, son aspectos que influyen positivamente en sus clientes para elegirlos. Para el $55 \%$ de ellos, ofrecer el crédito no es un factor determinante en la elección de sus tiendas como mejor opción de compra (Tabla 1). Este resultado concuerda con los expresado por Tovar Espitia y Mendoza Gómez (2009), quienes afirman que, en las tiendas de barrio, la existencia de factores estratégicos como la ubicación, la amabilidad, el crédito que otorgan a sus clientes, la dosis precisa del producto en una presentación de bajo desembolso, la amistad y el trato personalizado, han permitido continúen manteniendo una fortaleza integrando lo económico con lo cultural y comercial.

Otro aspecto a resaltar es el reconocimiento por parte de los tenderos de los gustos y preferencias sus clientes, el cual fue señalado por el total de los tenderos como un factor fácil de reconocer, y en éste influye el vínculo de amistad y la relación que establecen con ellos.

Tabla 1. Criterios que garantizan que un cliente compre en la tienda

\begin{tabular}{|l|c|c|c|c|c|}
\hline & PROXIMIDAD & PRECIOS & HORARIO DE ATENCIÓN & CRÉDITO & SURTIDO \\
\hline Muy en desacuerdo & $0 \%$ & $5 \%$ & $5 \%$ & $50 \%$ & $9 \%$ \\
\hline Desacuerdo & $0 \%$ & $5 \%$ & $0 \%$ & $5 \%$ & $0 \%$ \\
\hline Ni en acuerdo, ni en desacuerdo & $5 \%$ & $5 \%$ & $9 \%$ & $18 \%$ & $9 \%$ \\
\hline De acuerdo & $23 \%$ & $23 \%$ & $23 \%$ & $9 \%$ & $18 \%$ \\
\hline Muy de acuerdo & $73 \%$ & $64 \%$ & $64 \%$ & $18 \%$ & $64 \%$ \\
\hline
\end{tabular}

Fuente: Elaboración propia, a partir de cuestionario aplicado a tenderos de Manga

En lo que concierne a la relación tienda de barrio vs formatos comerciales de Hard Discount y Express se tiene que las siguientes ventajas que ofrecen estos formatos a los residentes del barrio Manga son:

- Cuentan con proveedores locales y extranjeros lo que asegura la variedad y calidad de productos, entre estas marcas que son propias.

- Productos a buen precio.

- Pagos en efectivo y con tarjeta.

- Cuentan con servicio de parqueadero.

- Están ubicados en zonas residenciales, escolares y empresariales. 
- Son negocios pequeños, pero bien organizados.

Los formatos comerciales Hard Discount y Express se caracterizan por su organización, ubicación en zonas estratégicas del barrio, prestan autoservicio a sus clientes, son elegidos para compras rápidas y los productos que ofrecen son atractivos por el precio, variedad en las marcas y pueden ser cancelados con diferentes medios de pagos.

Por lo general, los consumidores de esta clase de establecimientos son la población con niveles más bajos de ingresos económicos, lo que ha generado un gran reto para las cadenas de supermercados e hipermercados, ya que implica un cambio en la cultura ya construida desde hace muchos años y, el mejoramiento de sus estrategias de ventas, ofertas y precios. Estas superficies están definidas como negocios de ventas al detal con superficies de 100 metros cuadrados, con ventajas como la alta rotación, la proximidad y el trato personalizado con el cliente (Tiempo, 2004). Sin embargo, en la elección de las tiendas por parte de los clientes influyen criterios como la cercanía, los precios, horarios de atención y variedad de producto que ofrecen. Asimismo, los tenderos en la relación con sus clientes, reconocen sus gustos y preferencias, señalan, además, vínculos de amistad, un trato y servicio ofrecido considerado como muy bueno.

Es posible afirmar que la imagen de la tienda, recinto alegórico del barrio, aún se encuentra latente, por lo que es importante analizar entonces, qué consideran los tenderos sobre la llegada de los formatos Hard Discount y Express y de qué forma los han enfrentado, de tal suerte que sus tiendas, a pesar de los años, cambios socioeconómicos o del mercado, sigan más vivas que nunca. Para los tenderos, estos formatos que se han posicionado en su vecindario y, poco a poco, ha ido ganado más relevancia. Se sabe que han llegado al barrio, así como también, es posible identificarlos por sus nombres y determinar la resonancia con la que los habitantes del barrio hablan de ellos o, simplemente, los mencionan en uno u otro comentario. En este sentido, el nivel de amenaza que representa el haberse instalado con tanta rapidez y familiaridad, también es un aspecto que debaten los tenderos.

De este modo, dentro los formatos comerciales de Hard Discount y Express, que se sabe que hagan llegado al barrio, se encuentran Ara, Éxito Express, y Carulla. Así, el reconocimiento por parte de los tenderos, ya sea por visibilidad, renombre u otro aspecto, se puede percibir con mayor a menor 
posicionamiento en un $91 \%, 73 \%$, y $14 \%$ respectivamente ${ }^{3}$. El $64 \%$ de los tenderos de Manga reconocen que los formatos Hard Discount y Express llegaron a Manga para convertirse inmediatamente en su competencia directa y que, por tal razón, es una realidad que deben asumir. En este sentido, los encuestados aseguran que el nivel de amenaza que representa la llegada de estos formatos al barrio es medio (32\%) o alto (23\%). Vale anotar que una buena parte de los tenderos $(27 \%)$ consideran el nivel de amenaza es bajo.

Los aspectos en los que se concentra el mayor riesgo percibido por los tenderos, determinan la visión del nivel de amenaza. El precio, con un 68\%, la variedad con un 58\% y la calidad y presentación con un porcentaje igual de $54 \%$ para cada categoría, son los aspectos en los que más radica este riesgo. En contraparte, consideran que el horario de atención y la forma de pago, hacen parte de sus mayores fortalezas. Aquí cabe mencionar que el tema de la proximidad a las viviendas puede o no considerarse factores de riesgo; con un $45 \%$ en cada caso, el sondeo arroja que están de muy de acuerdo/de acuerdo y en muy en desacuerdo/desacuerdo igualmente, por lo que se puede decir que este elemento puede depender de circunstancias externas que indiquen el nivel de afectación dado (Tabla 2). No obstante, el $64 \%$ de los tenderos señalan que no ha sido necesaria la creación de nuevas estrategias para tal situación y que, más bien, puede hablarse de mejoramiento continuo y refuerzo constante.

Tabla 2. Factores en los que radica el riesgo del negocio por la llegada de los Formatos Comerciales Hard Discount y Express

\begin{tabular}{|c|c|c|c|c|c|c|c|c|}
\hline & $\begin{array}{c}\text { Horario de } \\
\text { atención }\end{array}$ & Proximidad & $\begin{array}{l}\text { Servicio al } \\
\text { cliente }\end{array}$ & $\begin{array}{c}\text { Forma de } \\
\text { pago }\end{array}$ & Presentación & Variedad & Calidad & Precio \\
\hline Muy en desacuerdo & $50 \%$ & $36 \%$ & $36 \%$ & $59 \%$ & $32 \%$ & $23 \%$ & $36 \%$ & $14 \%$ \\
\hline Desacuerdo & $9 \%$ & $9 \%$ & $0 \%$ & $0 \%$ & $14 \%$ & $9 \%$ & $0 \%$ & $14 \%$ \\
\hline Ni en acuerdo, ni en desacuerdo & $14 \%$ & $9 \%$ & $18 \%$ & $0 \%$ & $0 \%$ & $9 \%$ & $9 \%$ & $5 \%$ \\
\hline De acuerdo & $9 \%$ & $36 \%$ & $23 \%$ & $23 \%$ & $36 \%$ & $36 \%$ & $36 \%$ & $59 \%$ \\
\hline Muy de acuerdo & $18 \%$ & $9 \%$ & $23 \%$ & $18 \%$ & $18 \%$ & $23 \%$ & $18 \%$ & $9 \%$ \\
\hline
\end{tabular}

Fuente: Elaboración propia, a partir de cuestionario aplicado a tenderos de Manga

Los tenderos concluyen que el constante cambio de los precios, el factor tiempo, los clientes seguros o inclusive el no pensar en la situación como tal, serían las causas por las cuales no han sentido la necesidad de implementar nuevas estrategias. Las tiendas arraigadas se han mantenido haciendo lo mismo, apostándole siempre a la supervivencia y permanencia misma del negocio. Es decir,

\footnotetext{
${ }^{3}$ Los tenderos también consideran el mini-mercado La Esquina de Manga como formato Express. De hecho, un 5\% de los encuestados lo consideran así. Sin embargo, según la Cámara de Comercio de Cartagena, este negocio se encuentra registrado como tienda de autoservicio, por lo que no podría ubicarse entonces dentro del tipo de formato en mención.
} 
definitivamente no han recurrido a dichas tácticas, porque no ha sido necesario y no han sentido la llega de los formatos de Descuento duro y Express al barrio como una amenaza.

Tabla 3. Aspectos en los que se han reforzado estrategias para contrapesar el efecto de los Formatos Comerciales de Descuento duro y Express

\begin{tabular}{|c|c|c|c|c|c|c|c|}
\hline & $\begin{array}{c}\text { Horario de } \\
\text { atención }\end{array}$ & $\begin{array}{l}\text { Servicio al } \\
\text { cliente }\end{array}$ & $\begin{array}{c}\text { Forma de } \\
\text { pago }\end{array}$ & Presentación & Variedad & Precio & Calidad \\
\hline Muy en desacuerdo & $14 \%$ & $9 \%$ & $9 \%$ & $5 \%$ & $14 \%$ & $9 \%$ & $9 \%$ \\
\hline Desacuerdo & $5 \%$ & $5 \%$ & $0 \%$ & $0 \%$ & $0 \%$ & $0 \%$ & $0 \%$ \\
\hline Ni en acuerdo, ni en desacuerdo & $0 \%$ & $9 \%$ & $5 \%$ & $5 \%$ & $5 \%$ & $9 \%$ & $5 \%$ \\
\hline De acuerdo & $5 \%$ & $5 \%$ & $5 \%$ & $9 \%$ & $9 \%$ & $9 \%$ & $5 \%$ \\
\hline Muy de acuerdo & $18 \%$ & $23 \%$ & $9 \%$ & $18 \%$ & $18 \%$ & $18 \%$ & $27 \%$ \\
\hline No utiliza & $59 \%$ & $50 \%$ & $73 \%$ & $64 \%$ & $55 \%$ & $55 \%$ & $55 \%$ \\
\hline
\end{tabular}

Fuente: Elaboración propia, a partir de cuestionario aplicado a tenderos de Manga

Por su parte, el grado de efectividad de estrategias implementadas para enfrentar la competencia de los formatos comerciales Hard discount y Express, para los que han implementado algunas estrategias, representa un 54\% aprobadamente, entre bajo y muy bajo; para los tenderos de Manga la supervivencia de sus negocios, responde más a un tema de permanencia, antigüedad y, sobre todo, cercanía con los clientes. Sin embargo, por ello no se puede concluir que las tiendas no se encuentren preparadas para enfrentar cambios desde una perspectiva del micro o incluso macroambiente, sino que se puede decir más bien que para sus tenderos la mirada primordial es primeramente interna.

En efecto, las tiendas de barrio han asegurado su permanencia fortaleciendo el trato con los consumidores. Esta ha sido una de las principales características para que estos negocios se mantengan y los consumidores los sigan prefiriendo. Los tenderos del barrio Manga le apuntan conocimiento de los gustos y preferencias de sus clientes, con el fin de tener el inventario suficiente y, además, manejar las diferentes presentaciones que usualmente sus consumidores necesitan para sus compras diarias y asegurar proveedores que distribuyan productos de calidad.

Las nuevas superficies, evidentemente por su músculo financiero, y por tener una red de establecimientos que manejan prácticamente el mismo inventario de productos, aprovechan las economías de escala y compran unos volúmenes que les permiten trasladar las eficiencias en costos al consumidor para tener precios bajos, esto le da una clara ventaja frente a la tienda tradicional y así lo ha venido entendiendo el consumidor. Sin embargo, esto no quiere decir que los tenderos queden rezagados, esto podría llevar a que los tenderos de cierta manera, pudiesen agruparse, y generar una 
capacidad de volumen de compra que les permitiese obtener mejores precios y ser competitivos frente a estas nuevas superficies (Caicedo y Quiceno, 2015).

Sobre la necesidad de implementación de ayudas en las tiendas y sus tenderos para disminuir el efecto de la de la llegada los formatos comerciales Hard discount y express, el $50 \%$ considera que no es necesario apoyo técnico, mientras que el otro $50 \%$ si lo considera una ayuda oportuna. En este sentido, lo que, si consideran necesitar ayuda, piden recomendaciones sobre sobre precio, calidad y servicio al cliente con un $63 \%, 45 \%, 45 \%$ respectivamente.

\section{CONCLUSIONES}

El presente documento que tuvo como objetivo principal analizar las apreciaciones que tienen los tenderos de barrio Manga de Cartagena de Indias sobre los efectos socioeconómicos de la llegada de los nuevos formatos comerciales Hard discount y express al sector. Se llevó a cabo a partir de un estudio explicativo y propositivo de la percepción de los tenderos del barrio, involucrando la totalidad de las tiendas del sector.

Los resultados muestran que, en Cartagena de Indias, las tiendas de barrio tienen como ventaja un significado cultural que contribuye a la permanencia de las mismas en el mercado. Son proveedoras de productos de primera necesidad para las familias cartageneras y, además, tratan de acomodar los

precios de los productos requeridos por sus clientes. No obstante, frente a la contundente llegada de los formatos comerciales Hard discount y Express, si bien consideran que pueden ser una amenaza, gran parte de los tenderos no han considerado necesario implementar estrategias para fortalecer los factores que ellos califican como relevantes, mientras que quienes sí han ejecutado algún tipo de plan de acción, han tenido un nivel de efectividad bajo. Los tenderos señalan, sin embargo, la necesidad de recibir apoyo técnico con énfasis en factores como la calidad, precio y variedad que señalan, pueden poner en riesgo sus negocios en el futuro.

En este sentido se recomienda determinar o fortalecer planes de formación o estrategias de formación para los tenderos de Manga y asesorías técnicas específicamente sobre precio, calidad y servicio al cliente. Para ello, se espera que el acompañamiento sea de entidades tales como la Cámara de Comercio de Cartagena de Indias, UNDETCO- Agremiación de los tenderos- o de las Instituciones 
de Educación Superior, a partir de la labor social que realizan, en función del mejoramiento de la competitividad de los pequeños establecimientos como las tiendas de barrio. Asimismo, a partir de los resultados de este estudio, se podrá dar continuidad a futuras investigaciones que incluyan y relacionen el perfil del consumidor con las estrategias implementadas por tenderos y empresarios a gran escala.

\section{REFERENCIAS BIBLIOGRÁFICAS}

Acevedo Navas, C. y Páramo Morales, D. (2008). ¿Por qué las tiendas de barrio en Colombia no han fracasado frente a la llegada de las grandes cadenas de supermercados? Revista Entorno, 1. Retrieved from http://journalusco.edu.co/index.php/magazine/article/view/169/1975

Caicedo, J. y Quiceno, C. (2015). Situación Actual de la Tienda de Barrio Frente a la Aparición de las Nuevas Superficies ARA y D1 en la Ciudad De Manizales.

Castillo, L. V. y No, D. D. T. (2014). La Supervivencia de las empresas según indicadores empresariales. Modelo lineal mixto con datos de panel, periodo 2004 al 2008, caso de España. Working Papers 2014-13, Peruvian Economic Association

Londoño, E. y Navas, M. (2005). Canal tradicional en Colombia. Tiendas de barrio. Universidad Nacional de Colombia Sede Manizales.

Núñez, M. N. y López, H. E. (2012). Using the free association technique to know about the perception of the consumer of cheese from, 15(28), 271-286.

Paramo Morales, D. García Cano, O. L. y Arias Escobar, M. O. (2011). Hacia una tipología de tenderos de Manizales (Colombia). Pensamiento Y Gestión, (30), 93.

Pinilla, O. y González, G. (2004). Estudio comparativo de las características del merchandising aplicado la tienda tradicional de Barranquilla en estratos 2 y 5. Universidad del Norte. Barranquilla, Colombia. 
Ramírez, O. y Pachón, A. (2004). Una forma para entender la ciudad. Relaciones sociales y prácticas culturales en tres escenarios de mercado. Editorial Universidad Surcolombiana.

Ramos, O. S. (2016). Cuerpo y sentidos: el análisis sociológico de la percepción.

Tiempo, P. (2004). Minimercados se ponen de moda. Retrieved from http://www.eltiempo.com/archivo/documento/MAM-1567587

Tovar Espitia, S. A. y Mendoza Gómez, C. X. (2009). La importancia de la tienda de barrio como canal de distribución aplicado en la localidad La Candelaria, 65. Retrieved from http://repository.urosario.edu.co/bitstream/handle/10336/1051/1032364146-2009.pdf?sequence=1 